\title{
Methylphenidate produces selective enhancement of declarative memory consolidation in healthy volunteers
}

\author{
A. M. W. Linssen • E. F. P. M. Vuurman • A. Sambeth • \\ W. J. Riedel
}

Received: 31 July 2011 / Accepted: 30 November 2011 / Published online: 15 December 2011

(C) The Author(s) 2011. This article is published with open access at Springerlink.com

\begin{abstract}
Rationale Methylphenidate inhibits the reuptake of dopamine and noradrenaline and is used to treat children with attention deficit hyperactivity disorder (ADHD). Besides reducing behavioral symptoms, it improves their cognitive function. There are also observations of methylphenidateinduced cognition enhancement in healthy adults, although studies in this area are relatively sparse. We assessed the possible memory-enhancing properties of methylphenidate. Objective In the current study, the possible enhancing effects of three doses of methylphenidate on declarative and working memory, attention, response inhibition and planning were investigated in healthy volunteers.

Methods In a double blind placebo-controlled crossover study, 19 healthy young male volunteers were tested after a single dose of placebo or 10,20 or $40 \mathrm{mg}$ of methylphenidate. Cognitive performance testing included a word learning test as a measure of declarative memory, a spatial working memory test, a set-shifting test, a stop signal test and a computerized version of the Tower of London planning test.

Results Declarative memory consolidation was significantly improved relative to placebo after 20 and $40 \mathrm{mg}$ of methylphenidate. Methylphenidate also improved set shifting and stopped signal task performance but did not affect spatial working memory or planning.
\end{abstract}

A. M. W. Linssen $(\bowtie) \cdot$ E. F. P. M. Vuurman - A. Sambeth •

W. J. Riedel

Department of Neuropsychology \& Psychopharmacology, Faculty of Psychology and Neuroscience, Maastricht University, PO Box 616, 6200 MD Maastricht, The Netherlands

e-mail: anke.linssen@maastrichtuniversity.nl

W. J. Riedel

F. Hoffmann-La Roche Ltd,

Basel, Switzerland
Conclusions To the best of our knowledge, this is the first study reporting enhanced declarative memory consolidation after methylphenidate in a dose-related fashion over a dose range that is presumed to reflect a wide range of dopamine reuptake inhibition.

Keywords Methylphenidate $\cdot$ Dopamine $\cdot$ Declarative memory consolidation

\section{Introduction}

Cognition enhancement has received much attention in recent scientific literature due to our aging society and the increasing prevalence of Alzheimer's disease. However, the healthy young population also engages in drug use to enhance cognition as is illustrated by the abundant illicit use of drugs that are normally prescribed for the treatment of attention deficit hyperactivity disorder (ADHD) including methylphenidate (Ritalin) and other amphetaminelike stimulants, which are believed to improve cognitive performance (Greely et al. 2008b; Maher 2008). This has raised concern regarding the ethical and safety aspects of potential cognition-enhancing drugs (Greely et al. 2008a; Larriviere et al. 2009; Sahakian and Morein-Zamir 2007). These issues aside, it is important to know if these drugs do actually have cognition-enhancing effects in healthy subjects.

Methylphenidate inhibits the reuptake of the catecholamines dopamine and noradrenaline by blocking the transporters and thus, enhances catecholamine availability (Hannestad et al. 2010; Volkow et al. 1998). Methylphenidate treatment does not only reduce behavioral symptoms in children with ADHD but also improves their cognitive function (Pietrzak et al. 2006). There are also observations of methylphenidate-induced cognition enhancement in healthy adults (Elliott et al. 1997; Mehta et al. 2000), although studies in this area are relatively sparse. 
Previous studies on the effects of methylphenidate on cognitive function in healthy volunteers have mainly focused on cognitive domains which are known to be affected in ADHD or to involve dopaminergic action. These include attention, response inhibition, planning and (working) memory (Cools and Robbins 2004; Luciana and Collins 1997; Luciana et al. 1992; Mehta et al. 1999; Pietrzak et al. 2006). One aspect of attention studied in healthy volunteers is divided attention. Methylphenidate has been observed to improve performance on a divided attention task, in which participants have to track a moving target with a joystick and simultaneously press a button in response to the appearance of a visual stimulus (Bishop et al. 1997; Roehrs et al. 1999). These effects were most prominent when participants were sleep-deprived.

Attentional control has been assessed in healthy volunteers using an intradimensional/extradimensional shift task, which requires participants to make visual discriminations while stimulus characteristics vary along different dimensions and the relevant dimension reverses between different task stages (Rogers et al. 1999). Although Elliott et al. (1997) found no effect of methylphenidate on this task, Rogers et al. (1999) observed an enhanced ability to reallocate attention towards newly relevant features of environmental stimuli after methylphenidate.

Response inhibition, as assessed in a Go-NoGo task has been shown to improve with methylphenidate in older adults (Ben-Itzhak et al. 2008). Planning is also observed to be affected by methylphenidate in healthy adults. In two versions of the Tower of London task, enhanced performance was observed when methylphenidate was administered in the first of two sessions as opposed to impaired performance when the drug was taken in the second session (and placebo in the first, (Elliott et al. 1997)). Elliott et al. explain this result hypothesizing that methylphenidate enhances performance on novel tasks but impairs performance on familiar tasks.

There are several reports of improved performance on both nonspatial and spatial working memory tasks after methylphenidate administration in healthy adults. For example, methylphenidate enhances performance on the Sternberg memory scanning task, in which participants are asked to judge whether probe letters were in the memory set that was learned at the beginning of the task (Brumaghim and Klorman 1998; Fitzpatrick et al. 1988). Improvement of spatial working memory has been observed within a self-ordered search task in which participants search for tokens in an array of boxes displayed on a computer screen (Elliott et al. 1997; Mehta et al. 2000).

Besides the domains described above, few researchers have studied the influence of methylphenidate on immediate and delayed recall of word lists (Bray et al. 2004; Camp-Bruno and Herting 1994; Hermens et al. 2007; Kuypers and Ramaekers 2005), none of whom found a significant effect. This is surprising as methylphenidate is known to enhance delayed recall of word lists in ADHD (Evans et al. 1986). The pharmacologically similar compound amphetamine has shown to improve consolidation and retention of word lists while leaving acquisition unaffected (Advokat 2010). Experiments from Soetens and Zeeuws (Soetens et al. 1995; 1993; Zeeuws and Soetens 2007) have shown that amphetamine facilitates verbal memory recall if it is active in the brain during the consolidation phase (Soetens et al. 1995). Additional experiments showed that the effect increases with longer presentation time of the words, lasts at least 3 days, is independent of retrieval and generalizes to recognition (Soetens et al. 1995; Zeeuws et al. 2010; Zeeuws and Soetens 2007).

Importantly, task procedures used by Soetens and Zeeuws were rather different from those used in the methylphenidate studies cited above. Hermens et al., Kuypers and Ramaekers and Bray et al. used word lists containing only 12 or 15 items. In combination with repeated presentations (three or four times), this may have led to a ceiling effect in performance. In line with this notion, using lists of 24 easy nouns that were presented only once, Camp-Bruno and Herting (1994) did observe improved performance in both immediate and delayed recall, although this effect was not significant.

The aim of the current study was to find out whether enhanced word recall could be observed after methylphenidate with a more optimal experimental design. In order to avoid possible ceiling effects suspected to have influenced the studies mentioned above, a 30-word learning test was used in the current study. Performance on the 30 word lists of the visual verbal learning task was tested in 19 healthy volunteers after 10,20 or $40 \mathrm{mg}$ of methylphenidate and placebo. To get a more complete view of methylphenidate's cognitive effects, not only extending previous findings but also putting current effects of methylphenidate on word learning into perspective, additional cognitive tasks were administered. These additional tasks were carefully selected to represent the most relevant cognitive domains, both with respect to the most common clinical application of methylphenidate (i.e., treatment of ADHD) and dopaminergic involvement. Hence, we included tasks assessing attention, response inhibition, planning and working memory.

The design of the current study, using three different doses of methylphenidate in a placebo-controlled within-subjects experiment allows examination of the a dose-response relationship for this drug and its effect on cognition. It addresses some methodological issues observed in other studies using only a single dose or comparing different doses in parallel groups (Elliott et al. 1997; Mehta et al. 2000). Dose-related effects of moderate to high doses of methylphenidate on memory consolidation were expected to be reflected by better delayed recall. Performance on the other tasks was also expected to improve after moderate to high doses of methylphenidate. Based on the effect of methylphenidate on psychomotor function, it was expected that improved performance on these tasks may mainly be reflected by faster response times. 


\section{Method}

Participants

Nineteen healthy male volunteers (mean age $=23.4, \mathrm{SD}=5.4$, range $=19-37$ ) were selected and paid to participate. Participants were recruited by means of local advertisements. Prescreening occurred using a medical history questionnaire and was followed by medical examination.

The main inclusion criteria were: between 18 and 40 years of age, body mass index between 18 and $30 \mathrm{~kg} / \mathrm{m}^{2}$ and righthandedness. The main exclusion criteria were history or presence of mental or physical disorders, consumption of more than 21 alcohol units per week or more than five caffeine-containing drinks per day. In addition, volunteers with polymorphisms of the CES1 gene indicative of being a poor metabolizer of methylphenidate were excluded. One participant was excluded based on this criterion.

All subjects gave written informed consent. The study was carried out in accordance with the Declaration of Helsinki and approved by the medical ethical committee of Maastricht University.

\section{Design}

The study was conducted according to a double-blind, placebo-controlled, four-way crossover design. Between the testing days, a period of at least $48 \mathrm{~h}$ elapsed, but generally, testing days were scheduled approximately 1 week apart. Each participant received one of four single treatments including placebo (PLA) and 10, 20 and $40 \mathrm{mg}$ methylphenidate (M10, M20 and M40) on each testing day. The order of the treatments was randomized following a Williams Latin Square design resulting in four different sequences.

\section{Visual verbal learning test}

This task is an adapted version of Rey's Auditory Verbal Learning Test (Lezak 1995). The VVLT was used to measure declarative memory. It included 30 words that were presented on a computer screen (stimulus presentation time and interstimulus interval were 1,000 ms, (Klaassen et al. 2002)). This presentation was repeated three times using the same sequence of words, each time followed by immediate free recall of all remembered words. Thirty minutes after immediate free recall of the final series, participants were subjected to a delayed recall test and a recognition test. During the latter, 30 words were presented, 15 of which were previously presented and another 15 that were new. Outcome measures of the immediate and delayed recall tests were total number of correctly recalled words during immediate recall (summed over three trials) and number of correctly recalled words during delayed recall. The dependent measures of the recognition test were median reaction time, measured in milliseconds and sensitivity $\left(A^{\prime}\right) . A^{\prime}$ is calculated as follows: $A^{\prime}=1-1 / 4[\mathrm{fr} / \mathrm{cr}+$ $(1-\mathrm{cr}) /(1-\mathrm{fr})]$, with $\mathrm{fr}=$ falsely recognized words and $\mathrm{cr}=$ correctly recognized words.

Spatial working memory task

The 'Object relocation' program was used as a spatial working memory task (Kessels et al. 1999; 2000). In this task, participants were required to relocate ten visual stimuli (small drawings of objects such as a car or a flower) that were presented on a computer screen in a $15-\mathrm{cm}^{2}$ sized square to their original location. The task consisted of two parts. In the first part, called 'object to position' or OTP, participants had $15 \mathrm{~s}$ time to memorize the locations of the objects and were asked to drag the objects to the correct positions, which were cued with black dots. In the second part, the combined (COM) part, participants had $30 \mathrm{~s}$ to memorize the locations and this time, there were no cues to indicate the previous positions. Participants were instructed to place the objects in their original position as accurately as possible. In both parts, there was no time limit for the relocation of the stimuli. The dependent variables were percentage correct for OTP and percentage correct, absolute error and positional fit for $\mathrm{COM}$. In the COM part, percentage correct was calculated such that relocation would be correct when the object was placed within $20 \mathrm{~mm}$ from the original location. Absolute error was the absolute distance in millimeters an object was relocated away from its original position. Positional fit measured the ability to remember the locations per se, independent from the ability to remember which object was in each location and computed as the best fit score yielding the smallest distance error for the stimulus as a whole.

\section{Set shifting task}

The set shifting task used in this study combined the methods of Müller and Townsend (Muller et al. 2007; Townsend et al. 2006). The task measured cognitive flexibility and the influence of reward. Participants were presented with a stream of auditory and visual stimuli including light and dark blue squares on a computer screen and high and low tones through headphones (stimulus presentation time was $150 \mathrm{~ms}$ and interstimulus interval was $850 \mathrm{~ms}$ ). Assignments so as to respond to the tones ('Hear trials') or the squares ('Look' trials) were interspersed with the stimuli and presented for $1,000 \mathrm{~ms}$. Participants were told to respond only to the dark blue square and the high tones, while ignoring the light blue squares and low tones. In half of the trials, a ' $€$ ' sign on the computer screen indicated that if they would perform very well (correct and fast responses), they would earn an additional monetary reward (awarded to the best performer in the study). The experiment contained six blocks, of which three were 'reward blocks' and three were 'nonreward blocks'. Each block contained 56 
stimuli and switching occurred after a variable number of trials, ranging between five and nine trials. The sequence of blocks was randomized between participants and sessions. After a block containing the reward stimuli, the participants received feedback on how well they performed. Participants were expected to respond faster in the reward than in the control conditions. Dependent measures were response times in correct trials and percentage of correct responses. These were calculated separately for the four different trial types: Look in the reward blocks; Look in the nonreward blocks; Hear in the reward blocks; and Hear in the nonreward blocks.

\section{Stop signal task}

The stop signal task was used to measure motor impulsivity and adapted from that used by Fillmore and Rush (2002). In this task, participants were required to respond to go signals and inhibit the response when a stop signal was presented. On a computer screen, the go signals were visually presented letters, A, B, C and D (stimuli were presented for $500 \mathrm{~ms}$ or until the participant responded; interstimulus interval was $1,500 \mathrm{~ms}$ ). The stop signal was the visual presentation of a '*' at one of the corners of the screen. Participants were instructed to respond as fast as possible with left (to A and C) and right (to B and D) index fingers. Stop signals were presented at delays of $50,150,250$, and $350 \mathrm{~ms}$. A single test consisted of 176 trials in which each of the four-letter stimuli was presented equally often. A stop signal occurred in 48 trials during a test. Dependent variables were the proportion of commission errors on stop trials and the reaction times on go and stop-signal trials (i.e., stop reaction time). Reaction time to stop-signal trials represented the estimated mean time required to inhibit a response. The method for calculating stop reaction time was taken from the race model of inhibitory control (Logan 1994). Stop reaction time was calculated by subtracting the stop-signal delay from the reaction time on go trials associated with the $n$th percentile of the reaction time distribution. The $n$th percentile corresponds to the percentage of commission errors (Logan 1994; Ramaekers et al. 2011).

\section{Tower of London}

Planning ability was assessed by a computerized version of the Tower of London task (Sobczak et al. 2002). On a computer screen, two arrays of differently colored balls (red, yellow and blue) on sticks were presented until the subject responded. The subject was requested to indicate the minimal number of steps necessary to rearrange the balls on the lower configuration to match the arrangement presented on the top half of the screen. The subjects had to count the number of moves and then respond by pressing the appropriate response button (two to six steps) as quickly as possible. The complexity of the task was dependent on the minimal number of steps in which the rearrangement could be achieved: two, three, four and five steps and sometimes six steps, to avoid guessing (only two to five are analyzed). Performance was indicated by the slope coefficient of the linear regression of the median response time as a function of the number of steps. In addition, percentage correct and response time calculated per number of minimally required steps were taken into account.

\section{Procedure}

The current study was integrated in a larger study on the effects of methylphenidate on the Contingent Negative Variation (Linssen et al. 2011). A training session in which the tasks were practiced took place within 2 weeks before the first testing day. Participants abstained from alcohol during the last $24 \mathrm{~h}$ prior to each testing day. On testing days, they were collected at their home and arrived at the lab in fasted condition at either $8.00 \mathrm{am}$ or 8:30 am. Inclusion and exclusion criteria were checked. Next, participants were given a standardized meal for breakfast at $80 \mathrm{~min}$ predose $(t=-80)$. At $t=-30$, stop signal, set shifting and spatial working memory tests were administered to get a baseline measurement. At $t=0$ participants ingested four capsules with water, which contained either PLA, M10, M20 or M40. The stop signal, set shifting and spatial working memory tests were administered again at $t=90$ and $t=270$. The verbal word learning test and the Tower of London were administered at $t=150$, followed by delayed recall and recognition testing of the words approximately $30 \mathrm{~min}$ later. At the end of the testing day, participants were returned to their home.

\section{Data analysis}

Data were analyzed using a repeated measures analysis of variance. Because one subject dropped out, there was a minimal deviation from complete balancing of treatment orders (order 1 = PLA, M10, M20, M40 (five participants), order 2 = M10, M40, PLA, M20 (five participants), order $3=$ M20, PLA, M40, M10 (four participants) and order $4=$ M40, M20, M10, PLA (five participants)). For this reason, treatment order was entered as a between-subjects factor. Since treatment consisted of different doses of the same drug, main effects of the drug were not evaluated. Instead, a priori planned comparisons between each dose relative to placebo were tested for significance, at $p<0.05$ using LSD correction.

\section{Results}

Visual verbal learning test

Mean values of dependent measures of the visual verbal learning task are presented in Fig. 1. Methylphenidate had 
Fig. 1 Means of dependent measures (a total number of correctly recalled words in immediate recall; $\mathbf{b}$ number of words recalled in delayed recall; c median response time in recognition test; $\mathbf{d}$ sensitivity of recognition test) of the word learning test after placebo and methylphenidate, 10, 20 and $40 \mathrm{mg}$ (PLA, M10, M20 and M40, respectively). Significant differences relative to placebo are indicated by an asterisk a

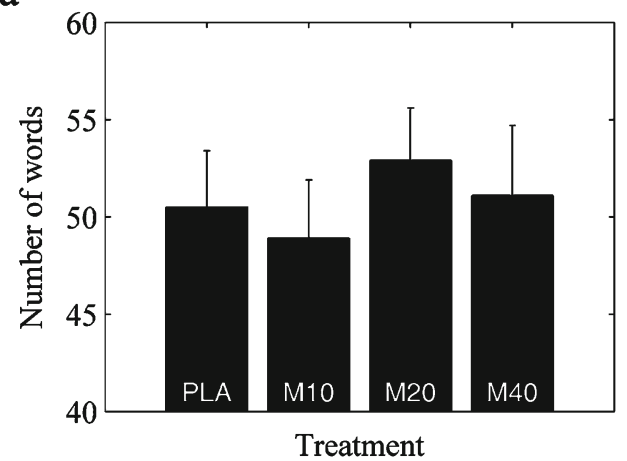

C

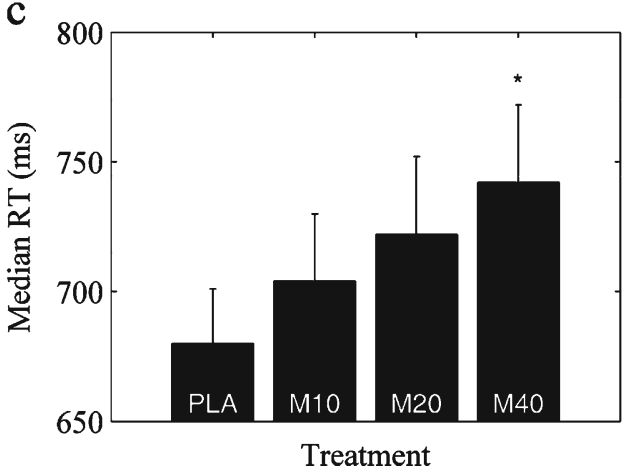

b

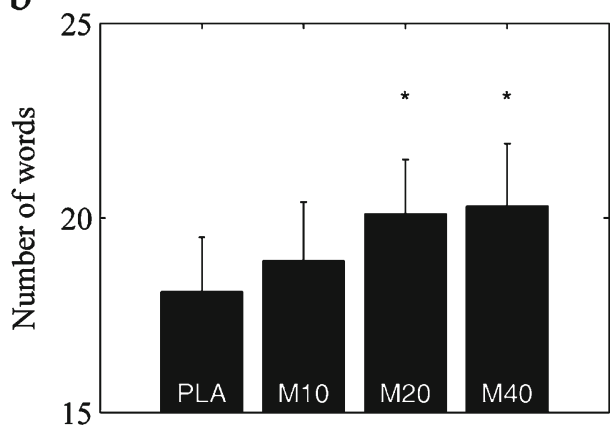

Treatment

d

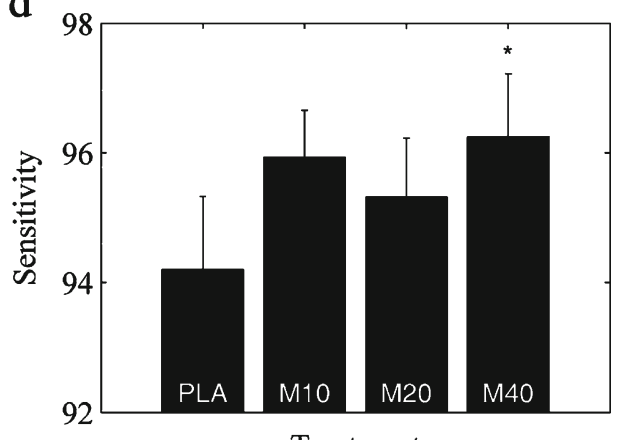

no effect on immediate recall. However, delayed recall improved after M20 $\left(F_{1,14}=4.937, p<0.05\right)$ and M40 $\left(F_{1,14}=6.084, p<0.03\right)$ compared to placebo.

Median reaction time on the recognition test was slower after M40 $\left(F_{1,14}=5.953, p<0.05\right)$. Sensitivity was higher after $40 \mathrm{mg}$ of methylphenidate $\left(F_{1,14}=4.878, p<0.05\right)$.

Spatial working memory task

Methylphenidate did not significantly affect spatial working memory task performance (Table 1).

Set shifting task

Methylphenidate did not affect response time and percentage correct in the 'look' trials in the reward blocks. In the nonreward blocks, there was also no effect of the drug on percentage correct. However, methylphenidate led to faster responses on the 'look' trials of the nonreward blocks after M40 at T90 $\left(F_{1,15}=9.589, p<0.01\right)$ and after M10 and M40, at T270 (M10: $F_{1,14}=5.363, p<0.04$, M40: $F_{1,14}=14.145, p<0.01$ ).

Responses to 'hear' trials were faster in the nonreward

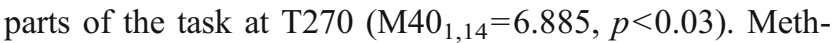
ylphenidate improved performance in the hear trials in both reward (M40: $\left.F_{1,15}=10.261, p<0.01\right)$ and nonreward trials (M20: $\left.F_{1,15}=4.713, p<0.05, \mathrm{M} 40: F_{1,15}=5.809, p<0.03\right)$ at T90 and reward trials at T270 (M40: $F_{1,14}=6.764, p<0.03$ ).
Stop signal task

A dose of $20 \mathrm{mg}$ of methylphenidate led to faster go and stop responses at T270 (go: $F_{1,14}=5.479, p<0.04$; stop: $\left.F_{1,14}=9.341, p<0.01\right)$. Performance on this task, as measured by percentage correct, was improved after M40 in stop trials at $\mathrm{T} 90\left(F_{1,15}=10.698, p<0.01\right)$.

\section{Tower of London}

Methylphenidate only positively affected performance of trials that could be solved in three steps when given at a dose of $20 \mathrm{mg}\left(F_{1,15}=5.065, p<0.05\right)$. Participants responded faster after M10 and in the trials that could be solved in five steps (M10: $\left.F_{1,15}=7.616, p<0.02\right)$ (Table 2).

\section{Discussion}

In this dose-response study, different doses of methylphenidate were tested for their effect on memory and other cognitive functions in healthy volunteers. Methylphenidate improved delayed recall of word lists. In addition, set shifting and stop signal test performance improved after methylphenidate. Methylphenidate had little effect on the Tower of London task performance and no effect on performance of the spatial working memory task. 


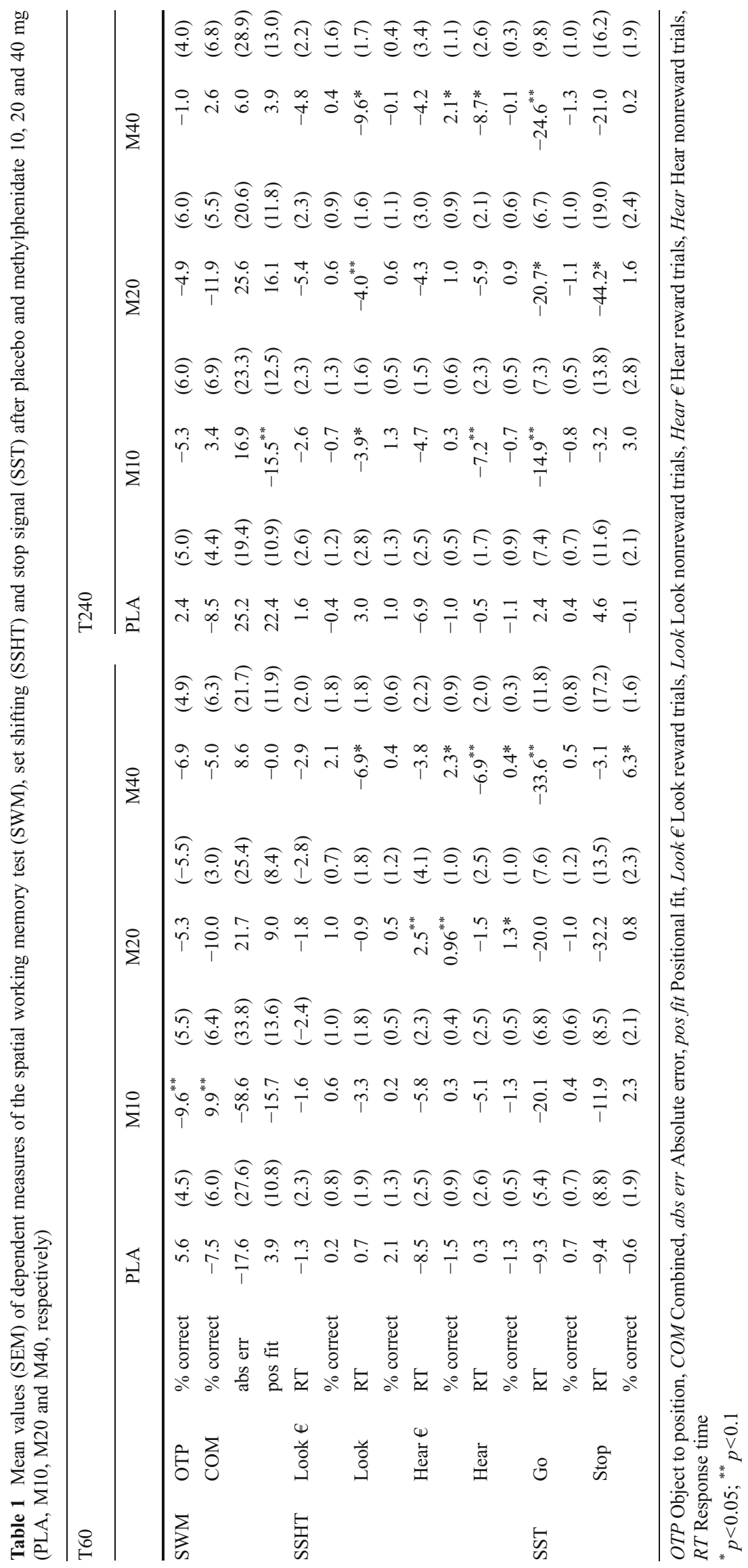


Table 2 Mean values (SEM) of dependent measures of Tower of London (ToL) after placebo and methylphenidate 10, 20 and $40 \mathrm{mg}$ (PLA, M10, M20 and M40, respectively)

$R T$ Response time ${ }^{*} p<0.05 ;{ }^{* *} p<0.1$
$\mathrm{T} 160$

\begin{tabular}{llcccc}
\hline & & \multicolumn{1}{l}{ PLA } & \multicolumn{1}{l}{ M10 } & \multicolumn{1}{l}{ M20 } & \multicolumn{1}{l}{ M40 } \\
\hline Slope & & $0.4(0.0)$ & $0.4(0.0)$ & $0.4(0.3)$ & $0.4(0.0)$ \\
2 steps & RT & $4,277(300)$ & $4,356(366)$ & $4,458(418)$ & $4,760(508)$ \\
& $\%$ correct & $95.8(2.3)$ & $95.9(2.1)$ & $95.1(1.6)$ & $94.9(2.3)$ \\
3 steps & RT & $6,039(502)$ & $6,461(333)$ & $5,835(446)$ & $6,128(583)$ \\
& $\%$ correct & $95.5(1.4)$ & $95.1(1.9)$ & $98.9^{*}(0.7)$ & $94.5(1.5)$ \\
4 steps & RT & $9,416(895)$ & $9,197(861)$ & $8,636(715)$ & $9,892(1,153)$ \\
& $\%$ correct & $91.6(2.3)$ & $89.6(2.1)$ & $93.5(2.2)$ & $94.3(2.7)$ \\
5 steps & RT & $13,458(1007)$ & $15,770(1,231)$ & $15,076(1,321)$ & $15,441(1,291)$ \\
& $\%$ correct & $84.1(3.3)$ & $85.3^{*}(3.0)$ & $79.8(2.7)$ & $85.4^{* *}(3.3)$ \\
\hline
\end{tabular}

Based on earlier findings of enhanced declarative memory consolidation after amphetamine, it was expected that methylphenidate would improve performance on the 30 word learning test. Results of the current study confirmed this expectation. Participants recalled more words in the delayed recall test after 20 and $40 \mathrm{mg}$ of methylphenidate compared to placebo. Since no improvement was observed at immediate recall, enhanced delayed recall is likely caused by better consolidation. To the best of our knowledge, we are the first to report better consolidation of word lists after methylphenidate in healthy adults. Since previous studies used similar doses (20 or $45 \mathrm{mg}$ ), suboptimal dosing is an unlikely explanation for the lack of performance-enhancing effects in those studies. An important factor may be word list length. When testing memory in healthy volunteers, a ceiling effect is easily reached if relatively short lists are presented repeatedly. Since it was hypothesized that enhanced dopamine activity induced by methylphenidate may lead to improved word learning test performance, the use of lists of 12 to 15 words in previous studies (Bray et al. 2004; Hermens et al. 2007; Kuypers and Ramaekers 2007) may have left no room for improvement.

There are ample indications of dopamine involvement in (spatial) working memory (Ellis et al. 2007; Landau et al. 2008; Robbins 2005). Indeed, methylphenidate has been shown to improve spatial working memory performance (Elliott et al. 1997; Mehta et al. 2000). This effect was, however, not replicated in the present study. A possibly important difference between the self-ordered search tasks used in previous studies and the spatial working memory task used here is that encoding of spatial locations occurs under time pressure in the current task. Previous research has suggested that methylphenidate may induce activity and arousal in healthy volunteers (Clark et al. 1986; Elliott et al. 1997). Hence, participants may have had difficulties to encode information due to overarousal.

Methylphenidate generally improved performance of the set shifting task, as was indicated by both faster responses and a higher percentage of correct responses. Most effects were observed on the hear trials, suggesting that methylphenidate affected auditory attention more than visual attention. It is unclear why this should be the case. Our expectation that the effect of methylphenidate would be more pronounced in the reward parts of this task was not confirmed. Participants may not have paid attention to the ' $€$ ' sign, which indicated that they were in the reward condition.

Methylphenidate improved stop signal task performance as was indicated mainly by faster responses. Effects were most prominent in the go trials. Faster responses (both in the set shifting and the stop signal task) may partly reflect the response readiness-enhancing effect of methylphenidate (Linssen et al. 2011) increasing the speed of performance (Elliott et al. 1997). Furthermore, taking into account the relatively high number of statistical comparisons being made in this study, some of the effects reported here including those on the Tower of London task may not be genuine drug effects. Therefore, the overall pattern of results was considered when describing drug effects. Thus, generally, MPH improved measures of declarative memory, attention and response inhibition.

The effects of methylphenidate were observed within a rather wide time window, between 90 and 270 min after drug intake. This likely reflects a relatively long lasting enhancement of dopamine levels in the blood after methylphenidate. Blood dopamine levels cannot be measured directly, but changes in prolactin level in blood are a good surrogate marker, since the prolactin level is known to rise as the dopamine level decreases and vice versa (Ben-Jonathan 1985). Indeed, prolactin levels were decreased between 60 and $240 \mathrm{~min}$ after methylphenidate administration in this group of participants (Linssen et al. 2011).

Most studies into effects of dopaminergic drugs on aspects of cognitive performance describe results according to an inverted $U$ curve when performance is mapped as a function of degree of neurotransmitter activity (Levy 2009; Mehta et al. 2004). The design of the present study, using 
three doses of methylphenidate, the highest of which corresponds to $75 \%$ blocking of dopamine reuptake sites (Volkow et al. 1998) should allow to reveal the inverted U curve, if present. This would be reflected by improved performance at low and intermediate dosing levels and a drop in performance enhancement at the higher dose. However, the current findings suggest that methylphenidate influences memory consolidation in a monotonic positive fashion. Although dose-response relationships may differ between cognitive domains, it has been shown that methylphenidate effects on response readiness cannot also be described in terms of the inverted $U$ function (Linssen et al. 2011). Methylphenidate's enhancing effects on response readiness were observed even after a dose as low as $10 \mathrm{mg}$ and grew more consistent and prominent with increasing doses. Hence, our data challenge the existence of the inverted U relationship, at least with respect to declarative memory consolidation and response readiness.

In conclusion, this was the first study to find dose-related effects of methylphenidate on declarative memory consolidation. This result is in line with earlier described findings of amphetamine-induced enhancement of memory consolidation. Improved performance was also observed on stop signal and set shifting tasks.

Acknowledgements This study was carried out at Maastricht University, Maastricht, The Netherlands and was sponsored by F. Hoffmann-LaRoche Ltd., Basel, Switzerland. WJR is an employee of F. Hoffmann-La Roche Ltd. and was involved in designing the study, analysis and monitoring of the data and approval of the report. The authors have full control of all primary data and agree to allow review of the data if requested.

The authors also would like to thank the following people for contributing to the study:

Dr. Cees van Leeuwen, Irene Lelieveld, Conny Quaedflieg, Saskia Schneider, Natalie Valle and Lizzy Vuurman.

The experiment complies with the current Dutch law.

Open Access This article is distributed under the terms of the Creative Commons Attribution Noncommercial License which permits any noncommercial use, distribution, and reproduction in any medium, provided the original author(s) and source are credited.

\section{References}

Advokat C (2010) What are the cognitive effects of stimulants medications? Emphasis on adults with attention-deficit/hyperactivity disorder (ADHD). Neurosci Biobehav Rev 34:1256-1266

Ben-Itzhak R, Giladi N, Gruendlinger L et al (2008) Can methylphenidate reduce fall risk in community-living older adults? A double-blind, single-dose cross-over study. J Am Geriatr Soc 56 (4):695-700

Ben-Jonathan N (1985) Dopamine: a prolactin-inhibiting hormone. Endocr Rev 6(4):564-589

Bishop C, Roehrs T, Rosenthal L et al (1997) Alerting effects of methylphenidate under basal and sleep-deprived conditions. Exp Clin Psychopharmacol 5(4):344-352

Bray CL, Cahill KS, Oshier JT et al (2004) Methylphenidate does not improve cognitive function in healthy sleep-deprived young adults. J Investig Med 52(3):192-201
Brumaghim JT, Klorman R (1998) Methylphenidate's effects on paired-associate learning and event-related potentials of young adults. Psychophysiology 35(1):73-85

Camp-Bruno JA, Herting RL (1994) Cognitive effects of milacemide and methylphenidate in healthy young adults. Psychopharmacology (Berl) 115(1-2):46-52

Clark CR, Geffen GM, Geffen LB (1986) Role of monoamine pathways in the control of attention: effects of droperidol and methylphenidate in normal adult humans. Psychopharmacology (Berl) 90(1):28-34

Cools R, Robbins TW (2004) Chemistry of the adaptive mind. Philos Transact A Math Phys Eng Sci 362(1825):2871-2888

Elliott R, Sahakian BJ, Matthews K et al (1997) Effects of methylphenidate on spatial working memory and planning in healthy young adults. Psychopharmacology (Berl) 131(2):196-206

Ellis KA, Mehta MA, Naga Venkatesha Murthy PJ et al (2007) Tyrosine depletion alters cortical and limbic blood flow but does not modulate spatial working memory performance or task-related blood flow in humans. Hum Brain Mapp 28(11):1136-1149

Evans RW, Gualtieri CT, Amara I (1986) Methylphenidate and memory: dissociated effects in hyperactive children. Psychopharmacology (Berl) 90(2):211-216

Fillmore MT, Rush CR (2002) Impaired inhibitory control of behavior in chronic cocaine users. Drug Alcohol Depend 66(3):265-273

Fitzpatrick P, Klorman R, Brumaghim JT et al (1988) Effects of methylphenidate on stimulus evaluation and response processes: evidence from performance and event-related potentials. Psychophysiology 25(3):292-304

Greely H, Sahakian B, Harris J et al (2008a) Towards responsible use of cognitive-enhancing drugs by the healthy. Nature 11:702-705

Greely H, Sahakian B, Harris J et al (2008b) Towards responsible use of cognitive-enhancing drugs by the healthy. Nature 456 (7223):702-705

Hannestad J, Gallezot JD, Planeta-Wilson B et al (2010) Clinically relevant doses of methylphenidate significantly occupy norepinephrine transporters in humans in vivo. Biol Psychiatry 68 (9):854-860

Hermens DF, Cooper NJ, Clark CR et al (2007) An integrative approach to determine the best behavioral and biological markers of methylphenidate. J Integr Neurosci 6(1):105-140

Kessels RP, Postma A, de Haan EH (1999) Object relocation: a program for setting up, running, and analyzing experiments on memory for object locations. Behav Res Methods Instrum Comput 31 (3):423-428

Kessels RP, Postma A, Wester AJ et al (2000) Memory for object locations in Korsakoff's amnesia. Cortex 36(1):47-57

Klaassen T, Riedel WJ, Deutz NE et al (2002) Mood congruent memory bias induced by tryptophan depletion. Psychol Med 32 (1):167-172

Kuypers KP, Ramaekers JG (2005) Transient memory impairment after acute dose of $75 \mathrm{mg}$ 3.4-methylene-dioxymethamphetamine. J Psychopharmacol 19(6):633-639

Kuypers KP, Ramaekers JG (2007) Acute dose of MDMA (75 mg) impairs spatial memory for location but leaves contextual processing of visuospatial information unaffected. Psychopharmacology (Berl) 189(4):557-563

Landau SM, Lal R, O’Neil JP et al (2008) Striatal dopamine and working memory. Cereb Cortex

Larriviere D, Williams MA, Rizzo M et al (2009) Responding to requests from adult patients for neuroenhancements: guidance of the Ethics, Law and Humanities Committee. Neurology 73 (17):1406-1412

Levy F (2009) Dopamine vs. noradrenaline: inverted-U effects and ADHD theories. Aust N Z J Psychiatry 43(2):101-108

Lezak MD (1995) Neuropsychological assessment, 3rd edn. Oxford University Press, New York 
Linssen AM, Vuurman EF, Sambeth A, et al (2011) Contingent negative variation as a dopaminergic biomarker: evidence from doserelated effects of methylphenidate. Psychopharmacology (Berl)

Logan GD (1994) On the ability to control thought and action: a user's guide to the stop signal paradigm. In: Dagenbach D, Carr TH (eds) Inhibitory processes in attention, memory, and language. Academic Press, San Diego

Luciana M, Collins PF (1997) Dopaminergic modulation of working memory for spatial but not object cues in normal humans. J Cogn Neurosci 9(3):330-347

Luciana M, Depue RA, Arbisi P et al (1992) Facilitation of working memory in humans by a D2 dopamine receptor agonist. J Cogn Neurosci 4(1):58-68

Maher B (2008) Poll results: look who's doping. Nature 452(7188):674-675

Mehta MA, Sahakian BJ, McKenna PJ et al (1999) Systemic sulpiride in young adult volunteers simulates the profile of cognitive deficits in Parkinson's disease. Psychopharmacology (Berl) 146(2):162-174

Mehta MA, Owen AM, Sahakian BJ et al (2000) Methylphenidate enhances working memory by modulating discrete frontal and parietal lobe regions in the human brain. J Neurosci 20(6):RC65

Mehta MA, Goodyer IM, Sahakian BJ (2004) Methylphenidate improves working memory and set-shifting in $\mathrm{AD} / \mathrm{HD}$ : relationships to baseline memory capacity. J Child Psychol Psychiatry 45(2):293-305

Muller J, Dreisbach G, Goschke T et al (2007) Dopamine and cognitive control: the prospect of monetary gains influences the balance between flexibility and stability in a set-shifting paradigm. Eur J Neurosci 26(12):3661-3668

Pietrzak RH, Mollica CM, Maruff P et al (2006) Cognitive effects of immediate-release methylphenidate in children with attention-deficit/ hyperactivity disorder. Neurosci Biobehav Rev 30(8):1225-1245

Ramaekers JG, Theunissen EL, de Brouwer M et al (2011) Tolerance and cross-tolerance to neurocognitive effects of THC and alcohol in heavy cannabis users. Psychopharmacology (Berl) 214(2): 391-401
Robbins TW (2005) Chemistry of the mind: neurochemical modulation of prefrontal cortical function. J Comp Neurol 493(1):140-146

Roehrs T, Papineau K, Rosenthal L et al (1999) Sleepiness and the reinforcing and subjective effects of methylphenidate. Exp Clin Psychopharmacol 7(2):145-150

Rogers RD, Blackshaw AJ, Middleton HC et al (1999) Tryptophan depletion impairs stimulus-reward learning while methylphenidate disrupts attentional control in healthy young adults: implications for the monoaminergic basis of impulsive behaviour. Psychopharmacology (Berl) 146(4):482-491

Sahakian B, Morein-Zamir S (2007) Professor's little helper. Nature 450(7173):1157-1159

Sobczak S, Riedel WJ, Booij I et al (2002) Cognition following acute tryptophan depletion: difference between first-degree relatives of bipolar disorder patients and matched healthy control volunteers. Psychol Med 32(3):503-515

Soetens E, D'Hooge R, Hueting JE (1993) Amphetamine enhances human-memory consolidation. Neurosci Lett 161(1):9-12

Soetens E, Casaer S, D'Hooge R et al (1995) Effect of amphetamine on long-term retention of verbal material. Psychopharmacology (Berl) 119(2):155-162

Townsend J, Adamo M, Haist F (2006) Changing channels: an fMRI study of aging and cross-modal attention shifts. NeuroImage 31 (4):1682-1692

Volkow ND, Wang GJ, Fowler JS et al (1998) Dopamine transporter occupancies in the human brain induced by therapeutic doses of oral methylphenidate. Am J Psychiatry 155(10):1325-1331

Zeeuws I, Soetens E (2007) Verbal memory performance improved via an acute administration of D-amphetamine. Hum Psychopharmacol 22(5):279-287

Zeeuws I, Deroost N, Soetens E (2010) Verbal memory improved by D-amphetamine: influence of the testing effect. Hum Psychopharmacol 25(5):377-387 